\title{
On boundary condition in tunnels under partial saturation
}

\author{
P. Gerard, R. Charlier \& F. Collin \\ University of Liège, Department ArGEnCO, Belgium
}

\begin{abstract}
A new hydraulic boundary condition modelling the hydraulic transfers between porous medium and ambient atmosphere occurring during gallery excavations is described. It combines two modes of water exchanges in partial saturation: seepage and evaporation flows. Numerical simulations of a gallery excavation in dilatant geomaterial are carried out in isothermal conditions. The results show the influence of hydric boundary condition on the convergence of the gallery and the importance of the determination of vapour transfer coefficient between atmosphere and porous medium.
\end{abstract}

\section{INTRODUCTION}

Nowadays, the solution for the high level radioactivity waste lies in nuclear waste disposals in deep and low permeable geological layer. All the processes altering this natural barrier are thus crucial issues. An important topic concerns the development of a damaged zone (named EDZ) during the excavation of the galleries. The extent of the EDZ increases the permeability of the host formation and consequently the radionuclides migration as well. A correct numerical prediction of the coupled processes occurring during the excavation is therefore needed. For this purpose, the hydraulic boundary condition at the gallery wall has a deep influence on the response of the low-permeability dilatant geomaterial. Indeed the usual boundary condition (for the flow problem) during excavation is a progressive decrease of the pore pressure down to the atmospheric pressure at the end of the excavation. On one hand, such boundary condition can lead to unphysical pore pressure distribution. On the other hand, the relative humidity in the gallery is usually controlled through an "air conditioning system". This could be modelled by a decrease of the pore pressure down to the corresponding suction at the end of the excavation [Hoxha et al., 2004]. This boundary condition relies on the assumption of a quasi-instantaneous equilibrium between the gallery relative humidity and the wall pore pressure. This highlights the need of a more detailed expression of the water exchanges between air gallery and gallery wall. Two modes of exchange can occur: seepage flow and vapour flow. The seepage flows are liquid flows that tend to reduce the gallery wall pore pressure down to the atmospheric pressure. Vapour exchanges occur when the relative humidities of air gallery and rock mass are different. Several formulations of the vapour flows can be found in the literature, which usually assume that the flow is proportional to the difference of relative humidity [Anagnostou, 1995], vapour pressure [Zhongxhuan et al., 2004], the vapour potential [Kowalski, 1997] or the volumetric vapour mass [Ben Nasrallah \& Pere, 1998].

In this paper, the expression of the new flow boundary condition in isothermal conditions is first developed (Section 2). After, an example of the influence of the hydraulic boundary condition will be presented for the excavation and ventilation of a deep cylindrical cavity (Section 3 ), before the discussions and the conclusions.

\section{WATER AND VAPOUR EXCHANGES AT THE GALLERY WALL}

During the excavation processes, the pore pressure at the gallery wall is decreasing. After excavation, for long term predictions, we can consider that a thermodynamical equilibrium has to be reached between the air gallery and rock mass. The wall moisture has to be in equilibrium with the air humidity in the tunnel. Water and vapour exchanges take place at the boundary between gallery and rock mass.

Water exchanges in liquid phase can occur according to the difference of water pore pressure between rock mass and gallery. In some coupled phenomena like dilatancy, numerical responses with classical boundary conditions of the flow problem provide totally unphysical results as an injection of a huge amount of water in the medium during excavation. An unilateral flow condition is thus imposed in order to avoid water inflow into the rock mass: water outflows can only be created if pore pressure in the formation 
is higher than the air pressure in the gallery. Seepage flow $\bar{S}$ can be expressed as follows:

$$
\begin{cases}\bar{S}=\beta \cdot\left(p_{w}^{f}-p_{\text {atm }}\right)^{2} & \text { if } p_{w}^{f} \geq p_{w}^{\text {gal }} \text { and } p_{w}^{f} \geq p_{\text {atm }} \\ \bar{S}=0 & \text { if } p_{w}^{f}<p_{w}^{\text {gal }} \text { or } p_{w}^{f}<p_{\text {atm }}\end{cases}
$$

with $p_{w}^{f}$ and $p_{w}^{g a l}$ the water pressures respectively in the formation and in the gallery, $p_{a t m}$ the atmospheric pressure and $\beta$ a seepage transfer coefficient. This transfer coefficient should be as high as possible (penalty condition) in order to respect the seepage condition.

Vapour exchanges occur when a difference between relative humidities of air gallery and rock mass exists. Vapour inflows or outflows are physically possible. Several formulations of these fluxes can be found in the literature. Each of them is using a mass transfer coefficient which can be expressed as a function of the degree of saturation, the porosity or the air windspeed in the gallery [Dracos, 1980; Anagnostou, 1995; Zhongxhuan et al., 2004]. To obtain the evaporation flow, this mass transfer coefficient can be multiplied by the difference of relative humidity [Anagnostou, 1995], vapour pressure [Zhongxhuan et al., 2004] or vapour potential [Kowalski, 1997] between air gallery and the geological formation. As proposed by Ben Nasrallah \& Pere [1998], we choose to express vapour exchanges as the difference of volumetric vapour mass between the tunnel atmosphere and rock mass:

$\bar{E}=\alpha \cdot\left(\rho_{v}^{f}-\rho_{v}^{g a l}\right)$

with $\rho_{v}^{f}$ and $\rho_{v}^{g a l}$ volumetric mass respectively in the formation and in the gallery and $\alpha$ a vapour transfer coefficient.

The volumetric vapour mass is given by the following thermodynamic relationship:

$\rho_{v}=h \cdot \rho_{v, 0}$

where $h$ is the relative humidity and $\rho_{v, 0}$ the saturated vapour volumetric mass.

Relative humidity in porous medium is related to the suction by the Kelvin's law and saturated vapour volumetric mass is obtained by ideal gas law.

The saturated vapour pressure given by the experimental expression following [Collin, 2003]:

$p_{v, 0}=a \cdot \exp (-b / T)$

with $a=112659 \mathrm{MPa}$ and $b=5192,74$ for temperatures included between 273 and $373^{\circ} \mathrm{K}$.

On the basis of previous relations, the total flow $\bar{q}$ between air gallery and the geological formation is simply expressed as the sum of the seepage flow and vapour exchange flux, which can occur near tunnel surface:

$\bar{q}=\bar{S}+\bar{E}$

Because of permanent air ventilation of the galleries in the tunnel, we can consider that air relative humidity and volumetric mass in the tunnel are constant. Evaporation and seepage flows evolve thus according to the value of water pressure $p_{w}^{f}$ at the gallery wall (Fig. 1). Initially, if:

- rock mass humidity is higher than air gallery humidity $\left(p_{w}^{f} \geq p_{\text {atm }}>p_{w}^{g a l}\right)$.

Vapour exchanges take place from the geological formation to the gallery. Evaporation flow remains constant as long as rock mass is totally saturated $\left(p_{w}^{f}>p_{a t m}\right)$. When soil surface water pressure is lower than atmospheric pressure, the geological formation is desaturated and vapour exchanges decrease until the equilibrium between porous medium and ambient atmosphere is obtained. Seepage flow exists only if pore pressure at the gallery wall is higher than gallery pore pressure.

- rock mass humidity is lower than air gallery humidity ( $p_{w}^{f}<p_{\text {atm }}$ and $p_{w}^{f}<p_{w}^{g a l}$ ).

Evaporation flows take place from the tunnel atmosphere into the formation in order to re-saturate the rock mass. The saturation increases progressively to reach the gallery relative humidity level. On the other hand, no seepage flow occurs, because only gaseous exchanges exist.

In order to solve numerically in finite element code the field equations using this new boundary condition, a linear auxiliary problem can be defined following the ideas of Borja \& Alarcon [1995] and the field of unknowns is obtained through a Newton-Raphson scheme. The linear auxiliary problem is discretized

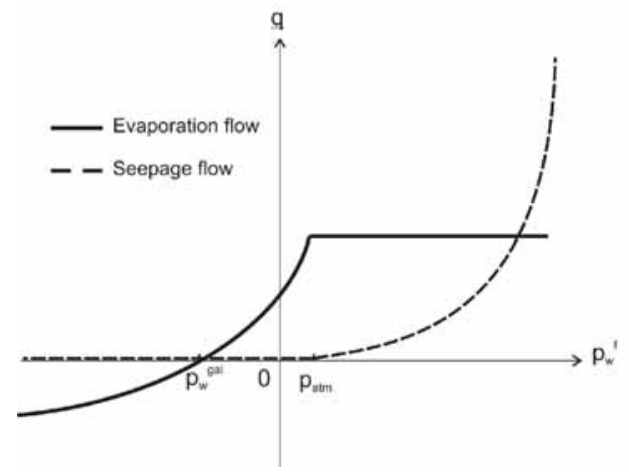

Figure 1. Evaporation and seepage flows. 
using the finite element methodology [Zienkiewicz \& Taylor, 2000]. Large strain isoparametric coupled finite elements and a specific element for the boundary condition have been introduced in the finite element code Lagamine [Collin, 2003] for the modelling.

\section{NUMERICAL MODELLING OF AN EXCAVATION}

Within the framework of nuclear waste disposals in deep geological layer, a correct numerical prediction of the coupled processes occurring during disposal excavations is needed. With the aim of studying the influence of hydric boundary condition, the excavation of a cylindrical gallery located in a homogeneous low permeability formation is simulated. The geometry and the mechanical law used are those proposed in the GdR-Momas benchmark exercise [Chavant \& Fernandez, 2005].

A cylindrical unsupported cavity of $3 \mathrm{~m}$ diameter is located in an homogeneous low permeability formation. The excavation process is modelled by decreasing the initial total stress and pore pressure towards atmospheric pressure. An initial isotropic stress state allows one dimensional axisymetrical modelling: $\sigma_{r}^{\prime}=7 \mathrm{MPa}$ and $p_{w}=5 \mathrm{MPa}$. Two steps are considered in the simulation: first the excavation process (duration $\mathrm{T}=1.5 \mathrm{Ms}$, around 17 days) and a second phase during which the radial convergence of the cavity evolves due to the water diffusion process. The final modelling time is $300 \mathrm{Ms}$ (about 9.5 years). At the external boundaries of our model, the initial conditions are assumed to be preserved in terms of total stress and pore pressure. This supposes that the external boundaries are far enough from the cavity. In the modelling, they are located at a radial distance seven times the cavity radius. This distance, maybe a little short to avoid boundary influence, is imposed by the geometry of the GdR-Momas benchmark.

The conditions are isotherms $\left(T=293^{\circ} \mathrm{K}\right)$ and gas pressure is assumed constant (equal to the atmospheric pressure).

\subsection{Mechanical constitutive law}

In order to reproduce the progressive decrease of the material strength, the elasto-plastic strain-softening model (with an associated Drucker-Prager yield criterion) proposed previously in the frame of GdR-Momas benchmark exercises [Chavant \& Fernandez, 2005] is used. Due to the associated plastic law, the resulting behaviour of the material is highly dilatant, which increases the coupling effects between the mechanical and the flow problem.

The following simulations have been performed with the parameters values defined in Table 1 .

\subsection{Hydraulic properties}

The mass flow $m_{i}^{t}$ is defined as follows:

$m_{i}^{t}=-\rho_{w}^{t} \frac{\kappa k_{r, w}^{t}}{\mu}\left(\frac{\partial p^{t}}{\partial x_{i}^{t}}+\rho_{w}^{t} g_{i}\right)$

where $\kappa$ is the intrinsic permeability, $k_{r, w}^{t}$ is water relative permeability and $\mu$ is the fluid viscosity.

The compressible fluid is assumed to respect the following relationship [Lewis \& Schrefler, 2000]. This predicts an increase of fluid density as a function of the pore pressure, defining $\chi_{w}$ as the fluid bulk modulus:

$\dot{\rho}_{w}^{t}=\frac{\rho_{w}^{t}}{\chi_{w}} \dot{p}^{t}$

The following parameters have been used in the excavation gallery simulation (Table 2 ).

The retention curve of the medium and the water relative permeability function are given by the following relationships, proposed previously in the frame of GdR-Momas benchmark exercises [Chavant \& Fernandez, 2005]:

$S_{r, w}=\left[1+\left(\frac{p_{c}}{10^{7}}\right)^{\frac{1}{1-0.412}}\right]^{-0.412}$ and $S_{r, w}=1$ if $p_{c}<0$

$k_{r, w}=\left[1+\left(S_{r, w}{ }^{-2.429}-1\right)^{1.176}\right]^{-1}$

with $S_{r, w}$ the water relative saturation, $k_{r, w}$ the water relative permeability and $p_{c}$ the capillary pressure $\left(p_{c}=p_{g}-p_{w}\right)$.

Table 1. Parameters of the mechanical model.

\begin{tabular}{llll}
\hline$E_{0}$ & Young modulus & 5800 & $\mathrm{MPa}$ \\
$v_{0}$ & Poisson ratio & 0.3 & - \\
$C_{0}$ & Initial cohesion & 1 & $\mathrm{MPa}$ \\
$\varphi$ & Friction angle & 25 & Degree \\
$\alpha$ & Residual cohesion & 0.01 & - \\
$\gamma_{R}^{p}$ & Dev. Strain threshold & 0.015 & - \\
\hline
\end{tabular}

Table 2. Parameters of the flow model.

\begin{tabular}{llll}
\hline$\kappa$ & Intrinsic permeability & $10^{-19}$ & $\mathrm{~m}^{2}$ \\
$\rho_{w, 0}$ & Water density & 1000 & $\mathrm{~kg} / \mathrm{m}^{3}$ \\
$\Phi_{0}$ & Initial porosity & 0.15 & - \\
$\chi_{w}$ & Bulk modulus & 2000 & $\mathrm{MPa}$ \\
$\mu$ & Dynamic viscosity & 0.001 & $\mathrm{~Pa} . \mathrm{s}$ \\
\hline
\end{tabular}




\subsection{Reference case}

In this axisymetrical modelling, a classical flow boundary condition is imposed: the pore pressures at the wall are decreased towards the atmospheric pressure during excavation and then remain constant (Fig. 2). Due to the hydro-mechanical coupling (dilatancy effect), a pore pressure decrease is observed in the damaged zone, which implies an unphysical 'numerical' injection of water into the formation.

Figure 3 presents the stress path followed in the first finite element at the wall. The behaviour is first elastic before the stress path reaches the initial yield surface. Due to softening, the cohesion is decreasing, inducing dilatancy at the same time. At the end of the modelling, the stress state tends to zero as no more deviatoric stresses are allowed. The radial displacement is equal to $1.75 \mathrm{~cm}$ at the end of the excavation and reaches $21.2 \mathrm{~cm}$ after $300 \mathrm{Ms}$. The coupling effects between the water diffusion and the mechanical process are thus important.

\subsection{Influence of hydraulic boundary condition}

The reference case highlights the need of a more detailed expression of the water exchanges between air gallery and gallery wall. Furthermore, relative

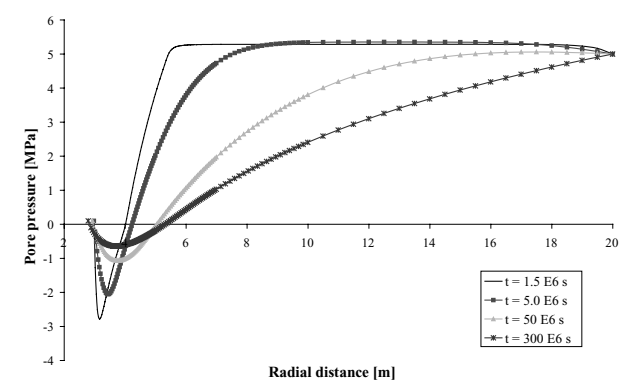

Figure 2. Case 1-Reference Case-Pore pressure distribution.

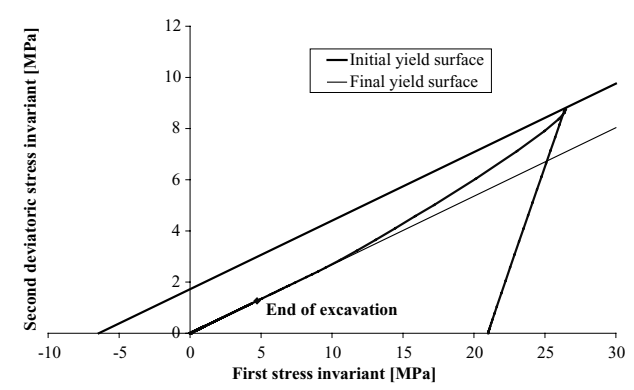

Figure 3. Case 1-Reference Case-Stress path curve. humidity in the tunnel is usually controlled by an "air conditioning system" maintaining constant air relative humidity. After excavation, for long term predictions, a thermodynamical equilibrium might be reached between the air gallery and the geological formation.

In these simulations (Case 2), a combined boundary condition with seepage and evaporation flows is thus used, as defined in Equation (4). A relative humidity of 0.96 (corresponding to a negative pore pressure of $-5 \mathrm{MPa}$ ) is imposed for the gallery atmosphere, but pore pressures at the wall are not controlled. The seepage transfer coefficient $\beta$ of Equation (1) is assumed equal to $10^{-7} \mathrm{~s}^{3} \cdot \mathrm{kg}^{-1}$.

The results depend on the vapour mass transfer coefficient $\alpha$, defined in Equation (1). However, this coefficient is difficult to determine. With a small vapour transfer coefficient (Case 2-1- $\alpha=$ $10^{-4} \mathrm{~m} / \mathrm{s}$ ), only seepage flows have influence on flow boundary behaviour. The pore pressure profiles (Fig. 4) tend towards atmospheric pressure on the wall and are thus similar to those from a simulation using only seepage boundary condition.

Using 100 times larger mass transfer coefficient (Case 2-2 $-\alpha=10^{-2} \mathrm{~m} / \mathrm{s}$ ), evaporation flow becomes preponderant on seepage flow. Pore pressure remains negative and close to the imposed pore pressure in

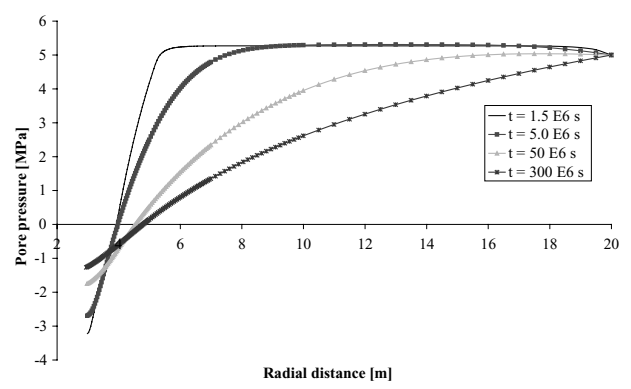

Figure 4. Case 2-1-Pore pressure distribution.

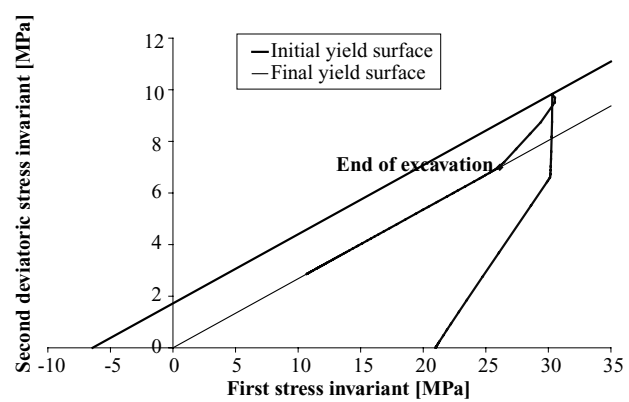

Figure 5. Case 2-1-Stress path curve. 


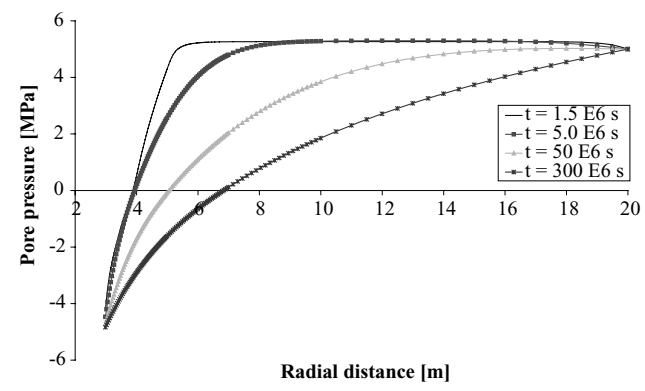

Figure 6. Case 2-2-Pore pressure distribution.

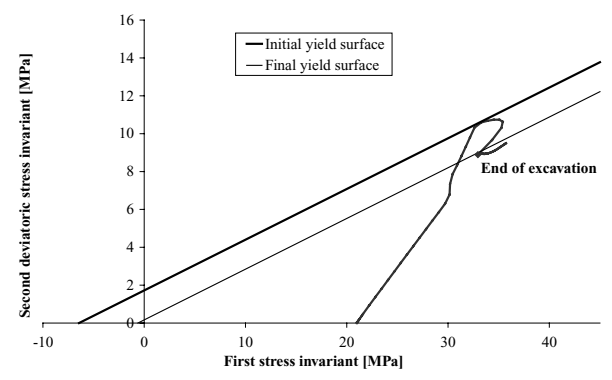

Figure 7. Case 2-2-Stress path curve.

the gallery (Fig. 6). The pore pressure profiles in the formation are quite similar to those obtained when a relative humidity (corresponding to a negative pore pressure of $-5 \mathrm{MPa}$ ) is imposed at the cavity wall as boundary condition.

Due to these different pore pressure distributions relative humidity at the wall evolves according to the vapour transfer coefficient. Figure 8 presents the temporal evolution of relative humidity of geological formation at the wall in different cases. With small vapour transfer coefficient (Case $2-1-\alpha=$ $10^{-4} \mathrm{~m} / \mathrm{s}$ ), seepage flow is predominant and the equilibrium between the gallery atmosphere and the wall is not reached at the end of the simulation. In the other hand, with high vapour coefficient (Case 2-2 $\alpha=10^{-2} \mathrm{~m} / \mathrm{s}$ ), the equilibrium is quickly reached. In an intermediate situation (Case 2-3 $-\alpha=10^{-3} \mathrm{~m} / \mathrm{s}$ ), seepage and evaporation flows are both influent. In a first time formation relative humidity increases, before decreasing to stabilize finally.

These different pore pressure distributions have a direct influence on the convergence predicted. Table 3 presents the results for the different cases. At the end of the excavation, the convergences are more or less the same. But as far as the long-term response is concerned, the predicted displacements are rather different. Indeed in Case 2-2, due to the high vapour transfer coefficient used, the remaining suction near

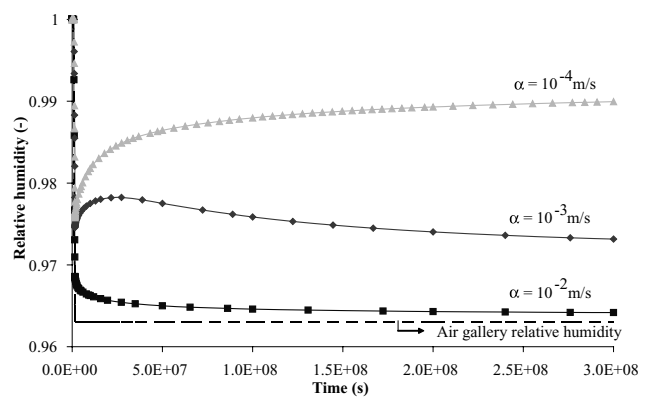

Figure 8. Case 2-Relative humidity evolutions.

Table 3. Cavity convergence for different cases.

\begin{tabular}{lllll}
\hline & Case 1 & Case 2-1 & Case 2-2 & Case 2-3 \\
\hline $1.5 \mathrm{Ms}$ & $1.75 \mathrm{~cm}$ & $1.50 \mathrm{~cm}$ & $1.41 \mathrm{~cm}$ & $1.48 \mathrm{~cm}$ \\
$3 \mathrm{Ms}$ & $21.2 \mathrm{~cm}$ & $5.28 \mathrm{~cm}$ & $1.47 \mathrm{~cm}$ & $1.73 \mathrm{~cm}$ \\
\hline
\end{tabular}

the tunnel ensures an additional strength and limits the material deformations. The stress paths followed in the first finite element near the wall confirm these results. Indeed, Figure 7 presents more or less the same stress states at the end of the excavation and after $300 \mathrm{Ms}$. The geological formation recovers an elastic behaviour at the end of the simulation and the high final value of the deviatoric stress is an indicator of the low plastic deformations. The comparison with the stress path in Case 1 (Fig. 3) shows clearly the difference of final value of the deviatoric stress and allows explaining the obtained convergences.

With small vapour transfer coefficient (Case 2-1), the stress path shows that the residual value of cohesion is reached and the behaviour is still plastic at the end of the simulation (Fig. 5). The final value of the deviatoric stress is a little higher than in Case 1, so that the convergence is less important. It is also interesting to note that the stress paths become purely deviatoric (constant mean stress) during excavation when atmosphere in the tunnel begins to be unsaturated, due to the expression of seepage flow (Eq. 1).

In the reference case (Case 1), the EDZ extends on 2.1 times the internal radius. With the mixed flow condition (Case 2-1/3), the simulations predict a rather narrow EDZ in comparison with the Case 1 . However, the EDZ in Cases 2-1, 2-2 and 2-3 are quite similar (between 1.71 and 1.74 times the internal radius), which means that the mass transfer coefficient has a small influence on the EDZ. The intensity of the corresponding plastic deformations is not the same, which involves the differences of convergence. 


\section{DISCUSSIONS AND CONCLUSIONS}

Within the framework of nuclear waste disposals in deep geological layer, a correct numerical prediction of the coupled processes occurring during theses excavations is needed. With the strain-softening constitutive model used, the coupling effects between water diffusion and the mechanical aspects are very important. A new boundary condition combining seepage and evaporation flows has been developed and the modelling has shown that the flow boundary condition at the cavity wall deeply influences the cavity convergence.

In low permeability and highly dilatant medium, wall pressure decreased (Case 1) leads to unphysical phenomenon, as the model predicts a massive injection of water into the formation. Furthermore thermodynamical equilibrium has to be reached between air gallery and rock mass, due to "air conditioning system" in the tunnel. This highlights the need of this new boundary condition, combining two modes of exchange: seepage flow and vapour flow. Depending on the value of vapour transfer coefficient, this boundary condition predicts low convergence, as for suction imposed condition or higher radial displacement as with only seepage flow condition. But with such coefficients, the computations provide realistic responses, that means 'physical' water flow and equilibrium between gallery atmosphere and rock mass relative humidities reached at the end of the simulation.

However, the value of the vapour exchange coefficient is difficult to determine. Experimental studies with clay sample will be realised to determine and analyze the influence of this coefficient.

\section{ACKNOWLEDGEMENTS}

The authors would like to thank the FRS-FNRS and the European project TIMODAZ for their financial support. TIMODAZ is co-funded by the European Commission (EC) as part of the sixth Euratom research and training Framework Programme (FP6) on nuclear energy (2002-2006).

\section{REFERENCES}

Anagnostou, G. 1995. Seepage flow around tunnels in swelling rock. Int. J. Numer. Anal. Meth. Geomech. 19:705-724.

Ben Nasrallah, S. \& Pere, P. 1998. Detailed study of a model of heat and mass transfer during convective drying of porous media. Int. J. Heat Mass Transfer 31-5:957-967.

Borja, R. \& Alarcon, E. 1995. A mathematical framework for finite strain elastoplastic consolidation part 1: balance law, variational formulation and linearization. Comput. Methods Appl. Mech. Engrg. 122:765-781.

Chavant, C. \& Fernandez, R. 2005. Evaluating the reliability of hydro-mechanical simulation: a benchmark of numerical techniques carried out by Research Group of MoMas. 2nd International Meeting Clays in Natural and Engineering Barriers for Radioactive Waste Confinement, Tours; 249-250.

Collin, F. 2003. Couplages thermo-hydro-mécaniques dans les sols et les roches tendres partiellement saturés. Thèse de doctorat. Université de Liège.

Dracos, Th. 1980. Hydrologie, Eine Einführung für Ingenieure. Springer-Verlag: Wien New York.

Hoxha, D., Giraud, A., Blaisonneau, A., Homand, F. \& Chavant C. 2004. Poroplastic modelling of the excavation and ventilation of a deep cavity. Int. J. Numer. Anal. Meth. Geomech. 28:339-364.

Kowalski, S.J. 1997. Moisture transport, thermodynamics, and boundary conditions in porous materials in presence of mechanical stresses. Chemical Engineering Science 52-7:1141-1150.

Lewis, R.W. \& Schrefler, B.A. 2000. The Finite Element Method in the Static and Dynamic Deformation and Consolidation of Porous Media. Wiley: New York.

Zhongxuan, L., Fengzhi, L., Yingxi, L. \& Yi, L. 2004. Effect of the environmental atmosphere on heat, water and gas transfer within hygroscopic fabrics. Journal of Computational and Applied Mathematics 163:199-210.

Zienkiewicz, O. \& Taylor, R. 2000. The Finite Element Method (5th edn). Butterworth-Heinemann: Stonchem, MA. 